\title{
Problemas
}

\section{epistemológicos \\ del concepto y definición de la psicología}

\section{Miguel Cruz Hernández}

\author{
Universidad Autónoma de Madrid
}

1. El tema de la definición de la psicología. ¿Es posible una definición siquiera sea aproximada de la psicología?. Me he hecho esta pregunta numerosas veces (1). Cierta. mente mi irrelevancia en el terreno de la psicología cientifica es evidente; y cuando las más destacadas figuras parecen esquivar el bulto o resuelven la faena con una larga cambiada, personalmente no tendría porqué inquietarme. Pero si el mal de muchos no es consuelo para nadie, tampoco el silencio de los más sabios debe servir de excusa a los aprendices.

Quienes en principio no se resignaron a esquivar este tema buscaron un primer ca. mino de aproximación: examinar lo que han dicho los psicólogos. Por desgracia esta ruta es tan larga como la de Ulises y con más Circes, Scilas y Caribdis que la recorrida por Odiseo. Los psicólogos, grandes o pequeños, actuales o del pasado, salvo muy contadas excepciones han operado en el tema mediante el socorrido recurso a la alusión y la elusión. En numerosos libros se alude a la psicología como saber a cerca de la conducta humana, del comportamiento del hombre, como antes se remitía a la ciencia de la mente o del alma humana, lo que solo sirve por lo común para eludir el problema, en tanto no existe un acuerdo a cerca de lo que sea la conducta, el comportamiento o la mente. Ciertamente Aris. tóteles sí sabia lo que era su 'u'yx́x una es. tructura asos formal eijoix propuesta para una potencia $\delta u v \chi \mu i$ corporal material. Y toda la Escuela -griega, judia, cristiana e islámica- lo conocía tambien en tanto utilizaba la terminología aristotélica, ya que no siempre su peculiar semántica.

No sucedió lo mismo con la concepción mentalista en tanto la mente fue definida por exclusión: lo que no era alma, más tam. poco reductible a la estructura orgánica corporal. En cuanto a la psicología actual, quienes no evitan el tema, se refieren a una conducta cuya precisión deja mucho que de. 
sear, salvo en los conductistas más estric. tos. No extrañe, pués, que se haya dicho que el término psicología no define nada; se trataría de un simple vocablo utilizado por los psicólogos para entenderse entre sí. Esto podria ser una mínima solución. Pero no nos hagamos ilusiones antes de haberla analizado

2. El recurso a la cuantificación del decir y hacer de lo psicólogos. Si el término psicología constituyera un punto de encuentro, cabria el recurso a la cuantificación, si no de lo que dicen, sí al menos de lo que hacen los psicólogos. Lo primero, ya ha sido cuantificado en excelentes trabajos. ¿Ha quedado algo claro? Sí: la no coincidencia en las opiniones, uno de los tropoi que citaban los grie. gos para probar la no posibilidad del saber:

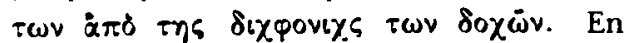
cuanto a lo segundo, el laberinto es aún más inestricable. Existen diferencias entre los quehaceres de psicobiólogos, psicomatemáticos, psicómetras, psicodiagnósticos, ecopsicólogos, etc.; pero incluso hay otras de carácter metodológico, estructural y teleológico, que incluso pueden darse dentro de los propios marcos regionales. Recuerde. se que algunos psicólogos se apoyan exclu. sivamente en parámetros de aprendizaje, o reducen este al animal; otros parten de la estricta estructura neuropsíquica, citoarquitectural o biomolecular; muchos en el pro. cesamiento de la conducta o de la psicoin. formación humana. Todo ello sin referir. nos a los pasatest o a los hábiles encubri. dores de ideologías más o menos psicologizadas, cuando aquellas solo pueden ser res. petables si se las declara abiertamente. En esta selva resultaría un azar encontrar, no ya un camino, ni una mala trocha que permitiese un tranquilo retorno.

¿Qué son, pués, las declaraciones o alusiones a un quehacer común que hacen los psicólogos? Si se piensa que los recursos sintácticos constituyen sin más una defini. ción, puede sestearse cómodamente. Pero a lo que intento referirme es a un concepto o definición lógicamente aceptable, que evite la conculcación de los viejos principios ya señalados en el Organon aristotélico: que lo definido no entre en la definición; que la especie esté limitada en el género por la di. ferencia específica; y que haya un propio de. terminado. Aplicados estos principios, la mayoría de las definiciones que encontra. mos se resuelven en pseudodefiniciones por silencio, copulación, remisión, elipsis superior y ex via remotionis. Voy a señalarlas muy escuetamente.

3. Pseudodefinición por silencio. La más usual de las pseudodefiniciones opera por "silencio". Consiste en ir recorriendo las definiciones más clásicas: etimológica, prin. cipalista, mentalista y conductista; se dese. chan por periclitadas las tres primeras y se invalida de iure la cuarta, en cuanto se le considera como incompleta, vaga y necesi. tada de una concreción posterior, que se anuncia y no se realiza, o no se justifica (2). En esta posición puede seguirse dos cami. nos: uno de talante más histórico y otro de presunción paradigmática. Para quienes siguen el primero, la psicología empezó sien. do la ciencia del alma, estructurada de acuer. do con un modelo principalista: la $\psi \dot{\psi} u \bar{x} \quad n e-$ fes, nafs, anima, alma), principio motor de las acciones peculiarmente humanas. Más tarde se convirtió en la ciencia de la mente, término éste último que releva al de alma, pero no al semema principalista. Finalmente, la psicología acabará siendo la ciencia de la conducta, con una matización sectorial arborescente sobre cuya frondosidad no es preciso insistir.

En cuanto al segundo camino, consiste en reducir el desarrollo histórico por una serie de paradigmas estruturales, unos peri. clitados (los principalismos anímico y menta lista), otros vigentes, unos más, algunos me nos: cibernetista, conductista, cognitivista, estructuralista, gestaltista, materialista dialectico, objetivista, psicoanalista, etc. Respecto al valor o utilidad de cada paradig ma, el autor concluye pro domo sua.

4. Las definiciones copulativas. Para obviar la ambigüedad del anterior sistema se 
ha recurrido a la definición copulativa, que consiste en resolver la dialéctica cerrada de la definición por silencio, generalmente en la presentación paradigmática, median. te una descripción completiva. La psicología sería así un saber a cerca del comportamiento humano (y) de su verificación desde distintos niveles, cognitivos, conductua. les, motivacionales, etc. Según el psicólogo se considere más o menos analista. cogniti. vista, conductista, dialéctico, ecopsicologis. ta, objetivista, etc. asi matizará la parte completiva unida por la conjunción copula. tiva. Las deficiencias lógicas de este sistema son evidentes; lo que se define no es la psi. cología científica sin más, sino la psicologia cognitivista, objetivista, etc., con lo cual una parte de lo definido entra en la definición. Sin embargo, es una postura cientificamen. te más honrada que la anterior.

5. La pseudodefinición por remisión. La remisión encubre un reduccionismo más o menos vergonzante; lo es menos cuando se trata de la remisión biologista y cibernética, lo es más en el caso de la eco-etologista. Me explicaré. Por poco que se sepa del tema, nadie niega la real existencia de unas bases biológicas en la psicologia; lo que se trata es de saber si lo psíquico se reduce a una manifestación epifenoménica de la reali. dad biológica, que solo puede admitir un tratamiento cuantitativo y unos modelos matemáticos, valiosos exclusivamente rebus sic stantibus; en cuanto se altere la modalidad cuantitativa, tambien hay que revisar la indole de los modelos. El juicio sobre la significación de los modelos cuantitativa. mente diseñados y llenados (almacenar, aprender, entender, percibir, recordar, sen. tir, tender, etc) como estructuras con reali. dad factual propia o estadística, queda aplazado o se le considera carente de sentido.

La peligrosidad de la remisión eco-etologista es doble. La primera tiene carácter coyuntural: el sex appeal del ecologismo, aunque en tanto novedismo su medicina es el tiempo; pero por ahora puede estropear a tantos alevines de psicólogos, como anta- ño al psicoanálisis y aún hoy el neo-jdeolo. gismo psicoanalítico, el neo-conductismo o la mania por las máquinas que tan aburri. dos hacen a los laboratorios, al decir de Skinner. La segunda proviene del radical riesgo de la circularidad eco-etologista: explicar el comportamiento en razón de un ámbito eco-etológico y a este último por un polisistema comportamental. Según por donde cortemos así resultará el color de la tajada; la más atrayente es la utópica.

6. La pseudodefinición por elipsis superior. Los reduccionismos biologista, cibernético y eco.etologista tambien encierran dentro el recurso a un nuevo tipo de pseudodefini. cón por elipsis superior, que el riesgo de la indefensión de la especie agrega la del género al que se remite. No solo nos quedamos sin saber lo que es psicología o comportamien. to, si no incluso lo que sea el mundo bioló. gico como ámbito de la conducta humana, o que es el hombre respecto a otras espe. cies vivas. Si el comportamiento es el estatuto de un ser vivo respecto de su medio y en cuanto este último a su vez puede ser modificado por aquel, no avanzamos un paso más allá del estricto saber natural: la. conducta es lo que hace el sujeto de experi. mentación, se trate del planaria o de un chimpacé, de las habilidades del perro del vecino o de lo que tan sabiamente hacen los profesores Brengelman, Eysenck, Luria o Skinner. Pienso que ninguno de los cua. tro citados aceptaría las consecuencias del anterior paradigma que nos deja sin saber lo que cientificamente es la conducta de un ser vivo cualquiera.

7. La definición ex via remotioniss. Las difi. cultades de las anteriores pseudodefiniciones han conducido a la utilización de la peligrosa ex via remotionis: se afirma lo que es la psicología negando todo cuanto vamos sabiendo que no es. Para ponderar su ries. go baste con señalar que el desarrollo de esta vía, pese a su actualísimo uso cibernético, procede de la dialéctica teológica. En el arduo problema de la índole del ser de Dios, cuya modalidad es en su raiz tan dis- 
tinta de la humana, la via analógica por matizada que sea, corre el peligro de antro. pomorfizar a Dios o de no poderle separar conceptualmente de la naturaleza como fuerza generadora. qúais . La via remotionis obvía el defecto de la equivocidad (nada podemos saber del ser de Dios) y el de la univocidad (el ser de Dios y el de lo creado no se diferencian sustancialmente). Que este método tenga tal origen no me parece mal; la teologia es un saber serio y un buen método de aprendizaje lógico hasta para los agnósticos y personalmente nunca le hice ascos. Pero la psicología no es Dios, gracias sean dadas a Allah; ni nada toto coelo diferente de la biologia, pongamos por ejemplo. Decir con la palabra hablada, o con la escrita, "la psicologia no es eso, ni aquello, ni lo de más allá), puede ser inicialmente todo lo científico y crítico que se quiera, pero a la postre resulta inútil y des. corazonador para los que se inician en este saber.

8. Psicología rin generes y psicología cientifica. El postulado científico. Ante el panorama alcanzado por el anterior análisis debemos preguntarnos como podemos encararnos con este problema. Para que el plantea. miento no resulte distorsionante conviene hacer una delimitación básica: la distinción entre la psicología in genere y la psicología cientifica. La primera tiene una extensión más amplia y general que la segunda y a ella pertenece el saber psicológico natural. Como he escrito hace más de veinte años "la conducta cotidiana del hombre está basada en un conocimiento práctico que cree calar -y que en realidad muchísimas veces penetra profundamente- en el carácter de nuestros semejantes y en su afectividad, de. seos y conocimientos. Por muy legos o muy leídos que seamos en psicologia, este conocimiento psicológico nos es tan connatural y tan necesario como el trato mismo con los seres humanos con quienes convivimos. Indudablemente este conocimiento varía mucho de unos hombres a otros... y se de. ben más exitos $-y$ más fracasos- a este conocimiento práctico del hombre, que todos los que pudieran proporcionar los más estupendos manuales de psicologia. La psicología (científica) pués, no quiere sustituir este conocimiento, intenta explicarlo... El psicólogo no debe olvidar la existencia de un psicología que se puede llamar profana o natural, pero que es extremadamente fructífera en el estudio de los caracteres. Esta psicología aparece esparcida en las grandes creaciones de los poetas, novelistas y autores dramáticos; $y$ aún en la vida de los diplomáticos, directores espirituales, etc.... Este observable puede enfocarse de dos modos diferentes: natural y científico. Respecto al primero... recomiendo la lectura reiterada de la novela y del teatro, consejo que me honro en compartir con Allport) (3). Por tanto, y aunque se aproveche la experiencia psicológica natural, el objeto a delimitar es el de la estricta psicología cien. tífica

¿Qué se exije a un saber para que sea considerado como científico?. Atenerse a una metodología previamente establecida que se apoya fundamentalmente en el mé. todo hipotético-deductivo. Por tanto, se pone entre paréntesis la supuesta «cosa en si) (res) se reduce el obiectum al estricto observable, y se exije que éste último lo sea en condiciones cuyo estatuto pueda ser homologado permanentemente dentro de una metodología previamente establecida ca. paz de cuantificación y verificación sistemá. ticas. La gran dificultad de estas exigenciạs reside eminentemente en la índole misma del observable de la psicología y en los re. quisitos necesarios para considerarlo como una real variable dependiente de otras au. ténticamente independientes.

El observable psicológico "científico» en tanto conducta coincide materialmente con el observable (natural), pero debe ser muy otro formalmente. La puesta entre parén. tesis del conocimiento conductual natural es condición necesaria pero no suficiente para la delimitación del ámbito de la psicología científica. Existe un saber conductual 
psicológico no estrictamente natural y sufi cientemente estructurado: el típico de la psicología filosófica. Nadie puede dudar que se apoya en la experiencia natural y en la observación empirica, pero rebasa el campo de la experimentación cualitativa y cuantitativamente apoyada en el método analítico-dialéctico. ¿Cuál, pués, es el terri. torio peculiar de la conducta científicamen. te considerada?: aquel que pueda ser verifi. cado en parámetros apriorísticamente esta. blecidos y sometidos a la estricta cuantificación. A este principio debe llamársele el primer postulado científico.

Aceptando el anterior postulado, la psicología científica se delimita como una cien. cia positiva física. Pero en tanto sus posibles fenómenos son peculiares del hombre, o de otras especies vivas, una concepción materialmente fisicalista reduciria la psicología cientifica a una parte de la fisiología.

9. La primera reducción conductista. Para no reducir la psicología cientifica a estricta fisiología, fue preciso aceptar una reducción: se trata de una auténtica ciencia positiva fisica, pero diferente de la fisiologia. Pero este principio es una reducción epistemológica, y como se evidencia con solo repasar los textos de psicologia, o con recordar el diálogo, un tanto de sordos, entre Eccles y Popper. Además, los psicológos por conductistas que sean, no renuncian ex propio ánimo ni al método analítico-dialéctico, ni a la recurrencia introspectiva; como tampoco los fisiólogos prescinden de la exploración estímulo-respuesta desde el estructuralismo citoarquitectónico o desde el funcionalismo biomolecular. Recuérdese como Skiner a la hora de analizar su peculiar conducta personal y cientifica en su Autobiografía, hace un estupendo uso, no solo de la intros. pección retrospectiva, incompatible con sus sistema científico, sino del análisis dialéctico. Afortunadamente lo que asi expone, con tanta gracia e ingenuidad, como profundidad y sabiduria, no tiene nada que ver con una hipotético registr' acumulativ's cien. tífico. Sin embargo, y pese a estos excursos, si la psicologia científica no es fisiología ni fi. losofía, solo le queda el territorio de la conducta entendida como un conjunto fenomé. nico positivo observable $y$ rerificable cuan. titativamente.

10. Segundo y tercer postulados conductistas. El concepto de conducta o comportamiento científicamente considerados es un nuevo postulado epistemológico. En puridad la conducta natural es fenoménica y positiva; lo que ahora se le pide es que sea observa. ble y verificable científicamente en pará. metros previamente establecidos. Naturalmente la conducta es fenoménicamente de ducible, lo que en términos físicos quiere de. cir estadísticamente pobable. Lo observa. ble y verificable científicamente son los es. tímulos y las respuestas, como Paulov y Watson sostuvieron con toda razón. Lo grave del caso es la amplitud de la supuesta caja negra, en la que se incluye un conjunto de mecanismos que cada vez son más y me. jor conocidos: los fisiológicos. Si no la conducta, al menos la relación estímulo-respuesta, opera en un organismo. Si se quiere obviar la consecuente reducción fisiologista se precisa de un nuevo postulado epistemológico: el análisis de la conducta o relación estímulo-respuesta debe ser realizado en utérminos de objeton, o sea, como hechos. Este es el más conflictivo de los postulados pre. sentados y pudiera pensarse que lo formulo en forma excesivamente filosófica. Pero no me pertenece su paternidad; está tomado de la obra de Skinner, The Behavior of Organism, publicada en 1938.

Las areducciones y los postulados neoconductistas. La critica de los anteriores principios epistemológicos constituye la casi totalidad de la historia de la psicología de los últimos setenta años. El análisis crítico del conductismo ha representado un papel decisivo en el desarrollo y evolución de la psicología actual; quien ésto escribe ha asumido desde muy mozo y con reiterada contumancia, dado lo limitado de sus cono. 
cimientos, la critica del conductismo integral. Aún así ningún psicólogo científico se ha atrevido a desmontar el término conducta. Lo que se ha hecho repetida y progresivamente es una limitación semántica del término mediante sucesivas precisiones. La primera reducción fue realizado por la pro. pia escuela conductista: la conducta como respuesta es una función del estímulo, verdad metodológica fundamental, pero que no aclara materialiter, pués explica lo que necesita precisión (la conducta) por un nue. vo concepto (función) no precisivo per sé.

Para resolver la anterior dificultad se concreta el problema en términos de variables los estímulos son la variable independiente; las respuestas, la dependiente; y entre unas y otras se admiten variables intermedias. Por tanto, las conducta como variable dependiente procede tanto de los estímulos (variables independientes) como del organismo (variables intermedias). Esta precisión, que en sus años aún jóvenes sostuvo Hull, encierra el peligro de la recurrencia al reduccionismo fisiologista; por lo cual en 1943 (Principios of Behavior) agrega a las variables intermedias fisiológicamente proce. sadas otras que en modo alguno son obser. vables directamente de un modo necesario y per sé; cuanto más serian deducibles. Esto conduce a un cuarto postulado, si no episte. mológico, sí metodológico: la conducta tiene una indole propositiva.

Skinner ha acusado con toda razón a las anteriores reducciones de desvirtuar el modelo conductista. Pese a la incidencia de Tolman en este tema (Purposive behavior in animals and men), no puede ser considerado como un estricto neo-conductista. Lo realizado por Tolman fue una nueva ureduc. cióny: la conducta es la relación significativa que se establece entre dos configuraciones estimulares, una de las cuales puede ser formalmente significativa. La explicación puede ser excelente para comprender las respuestas de anticipación, que pueden ser directas y cientificamente significativas. Pero explicar en términos de significación remite al contenido de la hipotética caja negra, lo cual no es admisible desde el punto de vista conductista. No es de extrañar, pués, que la siguiente precisión condujese a la resurrección del tema del sujeto psicoló. gico, la conciencia, que el conductismo habia empezado por desterrar. Es cierto que el neoconductismo sostiene que toda variable intermedia, aunque remita a la conciencia, es reducible a una situación "enmascarada" del sistema básico estímulo-respuesta. Pero en este caso el remedio es peor que la enfer. medad, como ya apareció en la curiosa po. lémica entre J. Herrnstein y Skinner (1977).

12. El postulado materialista-dialéctico y las reducciones emergentistas. Pareceria, pués, que el conductismo y el neo-conductismo deberian ser evitados en la precisión del concepto del psicología científica, en tanto el cognitivismo, el objetivismo y la psicolo. gia soviética no son reducibles al estricto paradigma conductual. Sin embargo, tam. poco estos últimos esquemas son menos vulnerables que el conductista. Todos ellos recurren a nuevos postulados o reduccio. nes epistemológicas, tan peligrosos o más que los conductistas. Para la psicología so. viética el postulado marxista: sólo existe una realidad esencial, la materia y el movi. miento, y una línea metodológica de valor no menos absoluto, el materialismo dialéc. tico. Hago gracia a esta posición de la posible acusación metafísica; pero aún reducida al carácter de un método de análisis, el a priori sigue siendo de un peso decisivo. $Y$ aunque la psicología soviética haya alcanza. do resultados científicos extraordinarios, este efecto pragmático no justifica episte. mológicamente su carácter científico, salvo si se admite el postulado marxista de la primacia de la praxis.

No menos peligrosa es la hipótesis emergentista en su cuádruple aspecto fisiologista, cibernetista, eco-etologista y psico-activista. Para el primero la auto-estructura subjetual conductual es el resultado actual del proce. so evolutivo que ha conducido al ser vivo 
hombre; la neuro-estructuración, la encefa. lización, la corticalización y la auto. estructura subjetual conductual son etapas del proceso evolutivo. Si esta evolución es meramente cuantitativa, volvemos al re. duccionismo fisiologista; y la psicología científica sería un efecto conductual alta. mente redundante de los mecanismos biomoleculares. Si por el contrario afirmamos que los fenómenos psíquicos no son reducibles a su origen filético neurofisiológico, re. caemos en el dualismo. Para el emergentis. mo cibernetista las operaciones, mecanismos o pautas supuestamente conductuales son circuitos de alta redundancia operados por un complejisimo y aún no del todo co. nocido sistema de ordenadores de medio liquido.

Para el primero, la auto-estructura subjetual conducta emerge del autosuficiente, auto. generable y autoregulable sistema ecoetológico, con lo cual la psicologia es un capitulo de una universal eco-etologia que funciona circularmente por sus complejos mecanismos de retroalimentación. Final. mente, para la cuarta posición, la autoes. tructura subjetual conductual, o la conciencia, si así gusta llamarla -y a mí no me desagrada- es una variable observable desde una formalización cognitiva, materialmen. te emergida de la realidad física, pero for malmente distinta de esta.

13. Elementos positivos en el análisis de la marcha dialéctica de la precisión del campo conductual ccientíficos. Podria parecer que este penoso recorrido a través de los intentos para precisar el territorio y la índole misma del campo conductual cientifico, debería concluir de un modo radicalmente negativo; no es esta mi opinión. Los postulados y reducciones epistemológicos son absoluta. mente necesarios en las ciencias formales y metodológicamente precisos en las ciencias físicas. Lo que se buscaba era la peculiari. dad (diferencia especifica) de la psicología científica. Y como sabemos bien, toda cien cia parte de un doble postulado: la posible existencia de un objeto que puede ser cien tíficamente considerado y de un método que proporcione validez matemáticamente significativa. Por tanto, la aceptación de postulados y reducciones no invalida totalmente la hipótesis de una posible psicolo. gía científica basada en la formalización con. ductual. Indudablemente, el objeto de di. cha ciencia no seria nunca la conducta secundum se, sino del modo como puede ser cientificamente considerada. El comportamiento humano es un todo, pero incluso como tal puede ser considerado dentro de muy diversos parámetros. En cuanto que pertenece a la vida del hombre, el compor. tamiento es físico. pertenece por su raiz a la naturaleza, y por su ámbito colectivo a la dimensión social. Por tanto, su estudio debe estar sometido a los principios gene. rales del método cientifico hipotético de. ductivo y a las técnicas metodológicas deri. vadas de aquel.

Ahora bien, el término conducta al que se refiere la psicología científica opera en el nivel de respuesta global a un modo pecu. liar del medio: en tanto éste actua como una situación estimular molar. Biofísica y biofisiológicamente la conducta se estudia de acuerdo con las leyes físico quimicas, a nivel general en el primer caso; de acuerdo con una sistemática orgánica estructural en el segundo. El nivel conductual propio de la psicología científica es posible en tanto los elementos biomoleculares han quedado formalizados en un conjunto de sistemas autogenerables y autorregulables estructu. rados molar y totalmente. Ciertamente una estructura asi concebida no permite esta. blecer una total solución de continuidad entre los distintos niveles científicos que materalmente se encuentran estrechamente conexionados; si cabe, empero, una distin. ción formal suficiente para determinar el objeto propio de la psicología. Por esto es preciso aceptar plenamente la segunda re ducción y al menos parcialmente la tercera.

Aunque a algunos parezca que una sim. ple distinción formal es suficiente, la lectura en términos de variables típica de la meto. 
dología científica psicologica tiene el suficiente apoyo objetual para considerarla con fundamento a parte rei. En ésto la psico. logia científica no es más ciencia que otras, pero tampoco menos. Recuerdese que todo organismo vivo es inseparable del te. rritorio natural y social en el que se desenvuelve y que esto alcanza a los fenómenos que estudia la biofísica y la química biomolecular y no se les niega el pleno estatuto científico de reales ciencias positivas delimitadas. Que el organismo vivo hombre no sea materialmente separable del territorio natural y social, no es un impedimento epistemológico para que furmalmente pue. dan delimitarse los diversos ámbitos de las ciencias sobre el hombre. De aquí el valor parcial que pueda tener el cuarto postulado epistemológico, indudablemente uno de los más "comprometidos»).

La ciencia no se delimita hoy tanto por el territono material al que se refiere, sino por el nivel formal a que opera. Que la con. ducta humana tomada en términos totales o de variables no sea realmente separable del territorio natural y social, no significa necesariamente que deba ser reducida a las estructuras biofisicas, biomoleculares, biocibernéticas u eco-etológicas (biosociales). Posiblemente ésto debe significar que el quinto postulado, la remisión a una hipóte. sis materialista-dialectica o las reducciones emergentista, deban ser cuidadosamente analizadas; su posible aceptación precede de instancias no epistemológicas, pertenecientes más al campo de la praxis que a la estricta teoría científica. Por ello me inclinaría a sustituirla por la sexta reducción, que permite una suficiente distinción for. mal.

El modo como se van reflejando los principios antes analizados en definiciones pue. de resumirse en el cuadro siguiente:

ESQUEMA DE LA MARCHA DIALECTICA DE UNA POSIBLE DELIMITACION DEL OBJETO DE LA PSICOLOGIA «CIENTIFICA» EN TERMINOS DE DEFINICION DESCRIPTIVA

POSTULADOS Y REDUCCIONES ESTIPULATIVAS CON PREMISAS ADICIONALES

1. Primer postulado: La ciencia es positiva.

2. Primera reducción: La psicología como ciencia no es reductible a estricta fisiología.

3. Segundo postulado: la conducta, objeto de la psicologia, es un conjunto fenoménico-positivo, observable y verificable en términos de estímulo-respuesta.

4. Tercer postulado: el análisis de la relación estímulo-respuesta debe ser realizado en términos de hechos ( = objeto).

\section{DEFINICIONES ACUMULADAS}

1. La psicologia es una ciencia positiva (del hombre).

2. La psicologia es una ciencia positiva distinta de la fisiología.

3. La psicología es la ciencia de la conducta como conjunto fenoménico-positivo, observable y verificable de la relación estímulo-respuesta.

4. La psicología es la ciencia de la conduc. ta como conjunto fenoménico positivo, observable y verificable de la relación estimulo-respuesta en términos de hechos dados (= objeto). 
5. Segunda reducción: los estimulos son considerados como variables independientes; las respuestas, como variables dependientes; entre unas y otras pucden existir variables intermedias.

6. Cuarto postulado: la conducta es expresable mediante proposiciones.

7. Tercera reducción: la conducta es la relación que se establece entre dos configuraciones estimulares, una de las cuales puede ser significativa.

8. Cuarta reducción: La relación conductual estimulo-respuesta remite a un sujeto.

9A. Quinto postulado: La raíz de la estructura conductual reside en el peculiar modo de la unica realidad material que se manifiesta real y dialécticamente.

9B. Quinta reducción A: La estructura conductual emerge del proceso evolutivo biológico, como efecto altamente redundante de los mecanismos biomoleculares.

9C. Quinta reducción B: La estructura conductual emerge como un sistema de circuitos de alta redundancia operados por un complejo sistema de ordenadores de medio liquido.

9D. Quinta reducción $C$ : La estructura conductal es uno de los términos de la relación ecoetológica.
5. La psicología es la ciencia de la conducta como conjunto de variables fenoménicas positivas, observables y verificables, dependientes tanto de los estímulos como del organismo.

6. La psicología es la ciencia de la conducta como conjunto proposicional de variables fenoménicas positivas, observables y verificables, dependientes tanto de los estímulos como del organismo.

7. La psicología es la ciencia de la conducta como conjunto proposicional de variables dependientes tanto de los estímulos como del organismo, en cuanto muestra una relación entre dos configuraciones estimulares, una de las cuales puede ser significativa.

8. La psicología es la ciencia de la conducta como conjunto proposicional de variables dependientes tanto de los estímulos como del organismo, en cuanto muestra una relación entre dos configuraciones estimulares, una de las cuales puede ser significativa y remite a un sujeto.

9A. (Posición de la psicología soviética).

9B. (Posición de la psicología biologista).

9C. (Posición de las teorias psicoinformáticas).

9D. (Posición eco-etologista) 
9E. Quinta reducción $D$ : La estructura conductal es una variable observable desde una formalización materialmente emergida de la realidad física, pero formalmente distinta de ésta.

10. Sexta reducción. La variable independencia es molar y se articula en un medio natural y social respectivo de ser vivo.
9E. La psicología es la ciencia de la conducta como conjunto proposicional de variables dependientes tanto de los estímulos como del organismo, en cuanto muestra una relación entre dos configuraciones estimulares, una de las cuales puede ser significativa y remite a una autoestructura subjetual observable a nivel cognitivo, emergida de la realidad física y formalmente distinta de ésta.

10. La psicología es la ciencia de conducta como conjunto proposicional de variables dependientes tanto de los estímulos como del organismo, en cuanto muestra una formalización de la interrelación respectiva del hombre y su medio natural y social.

11. Resumen: La psicología /cientifica/ es la ciencia de los principios, estructuras y funciones de la formalización conductual de la interrelación respectiva del hombre y su medio natural y social.

\section{Notas}

(1) La respuesta a esta cuestión es parte del correspondiente capitulo de un libro que un grupo de profesores de psicologiat estamos redactando y del que adelanto este resumen, en el que he prescindido del boato critico y bibliográlico.

(2) Este habría sido mi pecado como autor de un libro de psicologia. Que en él esté acompañado por muchos y relevantes psicólogos, no me exime de la correspon. diente penitencia.

(3) Lecciones de Psicologia. 4." edc. Edt. Revista de Occidente. Madrid, 1976. pp. 23. 117 y 120.

(4) Agradezco a mi compañero el profesor Dr. José Lorenzo González Fernández la lectura de las partes lógico-epistemológicas de este trabajo y las pertinentes obser. vaciones que me hizo. 